\title{
Interfacial Activity of new hydroxyl sulphonate betaine as surfactant for EOR
}

\author{
Chunfeng LIU
}

\author{
Chunfeng Liu No.5, Production Plant Of Daqing Oil Field, China
}

Keyword: hydroxyl sulphnate betaine; sacrificial agent; ultralow interfacial tension

\begin{abstract}
For the question of BS11 surfactant's poor deliquescence into consideration, the hydrophilic structural of amphibious surfactant is changed and the new kind of hydroxyl sulphonate betaine surfactant BS13 is developed. We researched that the interfacial tension,IFT, between the sewage solution of the new kind of hydroxyl sulphonate betaine surfactant BS13 and dehydrated crude oil from a wellhead in central district Xing-5,daqing is investigated, and the influences of the interfacial tension in one surfactant BS13 system by joining a little sacrificial agent Na3PO4 and polymer is studied. As a result the BS13 itself creates ultralow IFT in low mass fraction. With small amount of sacrifice, the interfacial tension system is more stable and the curve is more smoothly; some more amount of polymer is joined, the time in a low interfacial tension is longer and the system have better capacity in increasing the efficiency of oil washing in process of oil displacement.

Alkali-surfactant-polymer (ASP) ASP flooding is a kind of oil displacement technique which is relatively mature, good benefits in the tertiary oil recovery. In the practice of the field application in daqing oilfield, asp flooding can increase recovery efficiency about $20 \%{ }^{[1]}$. In field application, however, the selection of surfactant is imported more expensive alkyl benzene sulfonate ORS -41 . It affects the economic benefits of the asp flooding ${ }^{[2]}$. In addition, it appeared some problems in the industrial application of asp flooding.d.Due to the presence of alkali in ASP flooding, the problem is not only increasing polymer concentration, more important is greatly reduced the viscoelastic system At the same time, because of the presence of alkali, formed in the produced fluid of high viscosity of w/o emulsion not only affects the formation of oil well productivity, and greatly increasing the difficulty of demulsification. In the displacement liquid alkali, makes it difficult to process the effluence ${ }^{[3]}$, causes the formation of clay dispersion and migration, and leads to the decrease of the formation permeability. At the same time, alkali with reservoir fluid and rock mineral forms alkali scale ${ }^{[4]}$, damages the formation and affects the normal production of oil Wells ${ }^{[5]}$. Non-alkali binary compound surfactant - polymer flooding due to the biggest advantage of surfactant and polymer, when the chemical agent cost and asp flooding is under the same conditions, reached the same asp flooding oil displacement effect, and relatively simple asp flooding technology, and reservoir and oil well scaling, the advantages of easy processing effluence become one of the development direction of composite drive[6]. In SP flooding, the stability of surfactant containing non-alkali or weak base and daqing crude oil form $10-3 \mathrm{~mm} / \mathrm{m}$ ultra-low interfacial tension is the key ${ }^{[7] 8][9]}$.

Therefore, the author studied on the condition of alkali free new carboxyl betaine BSA interfacial tension. Indoor research results show that between BS system and daqing crude oil in a wide range of interfacial tension can reach 10-3 $\mathrm{mn} / \mathrm{m}$ orders of magnitude.For the second order ion salinity it has a strong ability to adapt. Because sodium phosphate is a kind of weak base more weak than sodium carbonate, and almost doesn't damage to formation, and in many cases, sodium phosphate was used as a sacrificial agent in the chemical flooding surfactants. As a result, we particularly studied the influence of different mass fraction on the interfacial tension sodium phosphate.
\end{abstract}

\section{The experiment}

(1) The experiment of crude oil:Daqing oilfield A station Wellhead dehydration of crude oil, flash-poin $60^{\circ} \mathrm{C}$, freezing point $38^{\circ} \mathrm{C}$, density $\left(20^{\circ} \mathrm{C}\right) 0.8591 \mathrm{~g} / \mathrm{cm}^{3}$, kinematic viscosity $50.49 \mathrm{~mm} 2 / \mathrm{s}$, 
paraffin content $21.07 \%$, gel content $21.47 \%$, sulfur content $0.072 \%$, carbon residue $2.73 \%$, ash content $0.04 \%$.

(2)With liquid water: Daqing oilfield A station recycled produced water, degree of mineralization $4063.4 \mathrm{mg} / \mathrm{L}, \mathrm{Ca}^{2+} 25.35 \mathrm{mg} / \mathrm{L}, \mathrm{Mg}^{2+} 4.20 \mathrm{mg} / \mathrm{L}$.

Table1 Daqing oil production A standing reinjection sewage of ions and salinity

\begin{tabular}{c|c|c|c|c|c|c|c}
\hline $\mathrm{CO}_{3}{ }^{2-}$ & $\mathrm{HCO}_{3}{ }^{-}$ & $\mathrm{CL}^{-1}$ & $\mathrm{SO}_{4}{ }^{2-}$ & $\mathrm{Ca}^{2+}$ & $\mathrm{Mg}^{2+}$ & $\mathrm{Na}^{2+}$ & $\begin{array}{c}\text { total mineralization } \\
(\mathrm{mg} / \mathrm{L})\end{array}$ \\
\hline 275.49 & 1851.65 & 602.82 & 27.62 & 25.35 & 4.20 & 1276.27 & 4063.40 \\
\hline
\end{tabular}

(3)Chemicals: analysis of pure Na3PO4, polymer, BS13 surfactant's poor.

(4)Experimental temperature: $45^{\circ} \mathrm{C}$.

(5)Experimental apparatus: The American Texas - 500 spinning drop interfacial tensiometer electronic balance, oven.

\section{Results and Discussion}

\subsection{BS13 surfactant mass fraction}

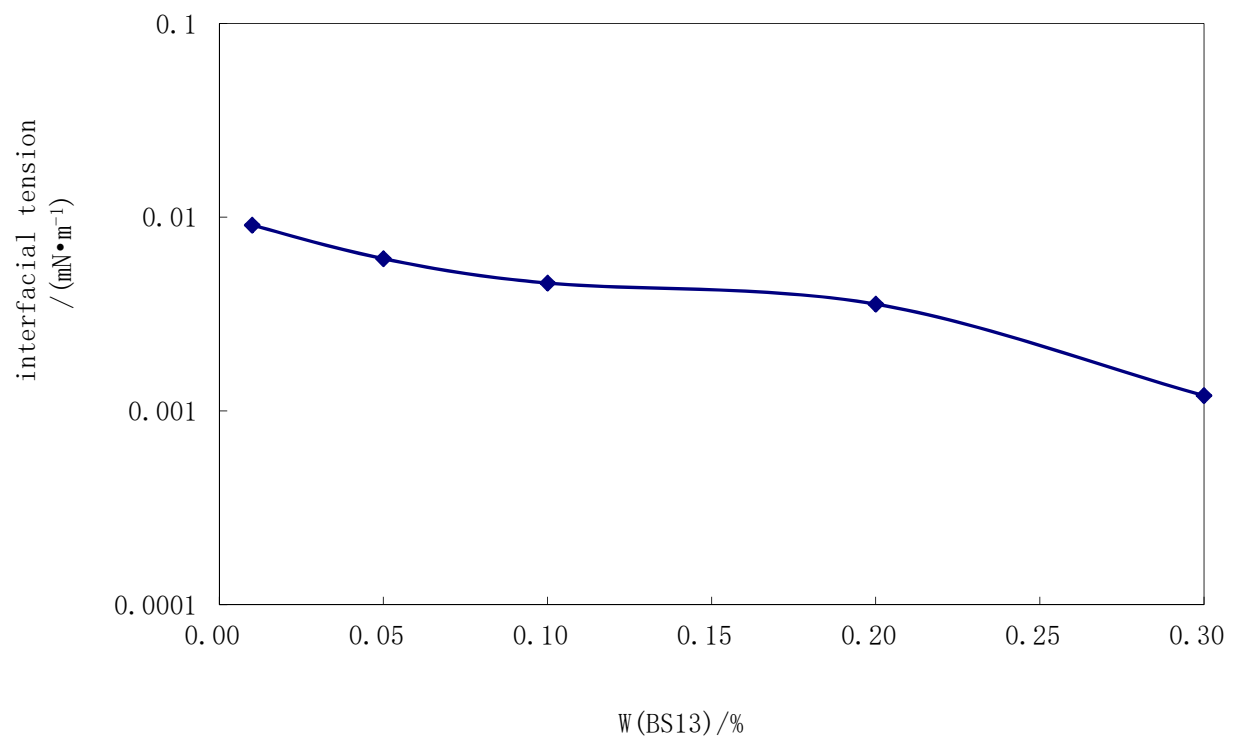

Figure 1 The influence of surfactants on the interfacial tension of crude oil in the different mass fraction of BSA

From figure 1, you can see that when the mass fraction of BSA increases, the interfacial tension of non-alkali BSA system is reduced, and when BSA mass fraction is very low $(0.05 \%)$, the system of ultra-low interfacial tension reached, at the same time, the mass fraction of ultra-low interfacial tension range is wide. That shows this kind of surfactants is very lower interfacial tension of the efficiency. 


\subsection{Sodium phosphate quality score}

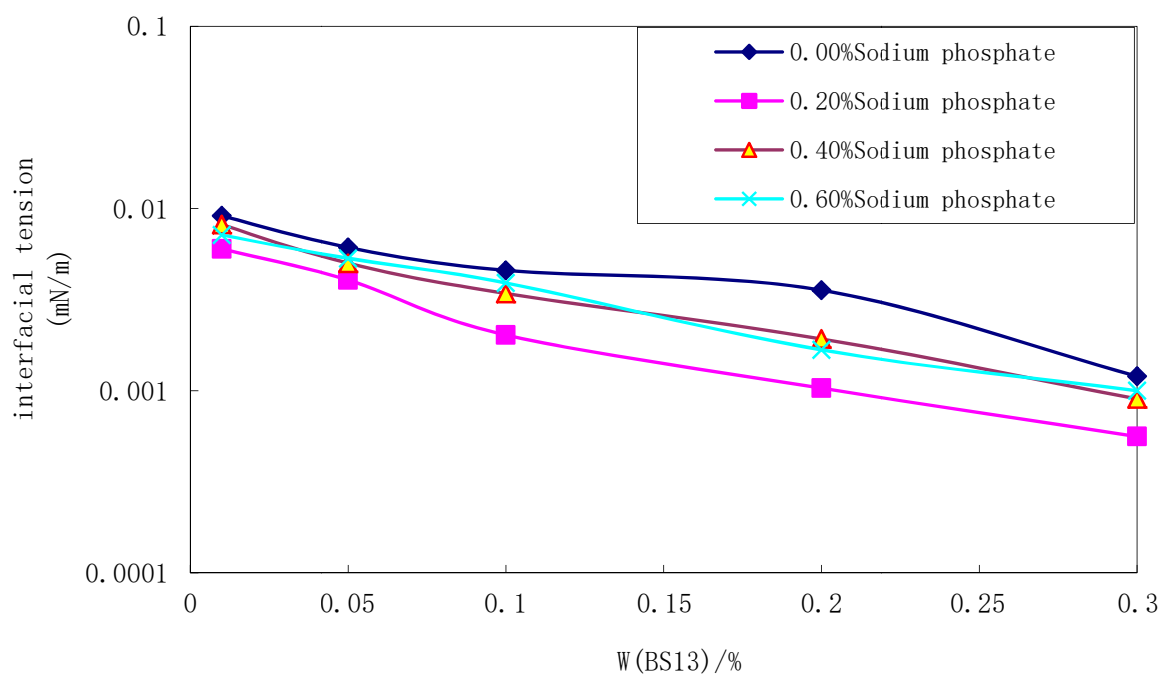

Figure 2 Adding different mass fraction of sodium phosphate, different mass fraction of BSA, the interfacial tension of surfactant system

Sodium phosphate mass fraction were $0.0 \%, 0.2 \%, 0.4 \%$ and $0.0 \%$ respectively, with different mass fraction of BSA, the interfacial tension of surfactant system change rules as shown in figure 2 , can be seen from figure 2, whether or not to join the sodium phosphate, when the mass fraction of BSA surfactant increases, the system of interfacial tension is reduced. And it is compared joining the curve of the sodium phosphate with not joining the curve of the sodium phosphate, adding sodium phosphate solution system of interfacial tension values significantly lower than the values of interfacial tension and sodium phosphate system, namely adding sodium phosphate can further reduce the interfacial tension of system. Can be seen from the figure 2 also, adding $0.2 \%$ sodium phosphate of interfacial tension is significantly lower than the $0.4 \%$ and $0.6 \%$ sodium phosphate of interfacial tension, That means adding certain mass fraction of sodium phosphate can make the system to achieve ultra-low interfacial tension, interfacial tension after more than the optimal value will not be reduced because of the mass fraction of sodium phosphate increase.

2.3 The influence of the interfacial tension of polymer of BSA surfactant - sodium phosphate system

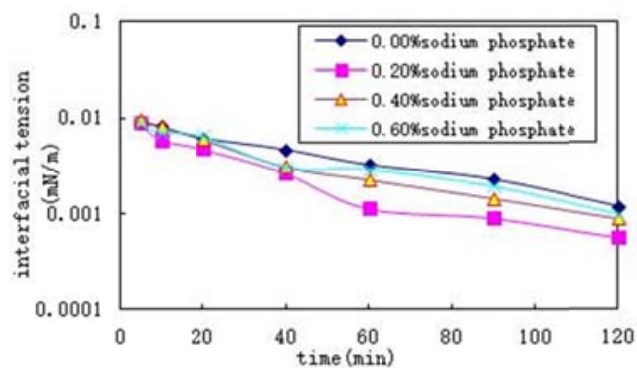

Figure 3 the dynamic interfacial tension $0.3 \%$ BS13 in different mass fraction of sodium phosphate solution (without polymer)

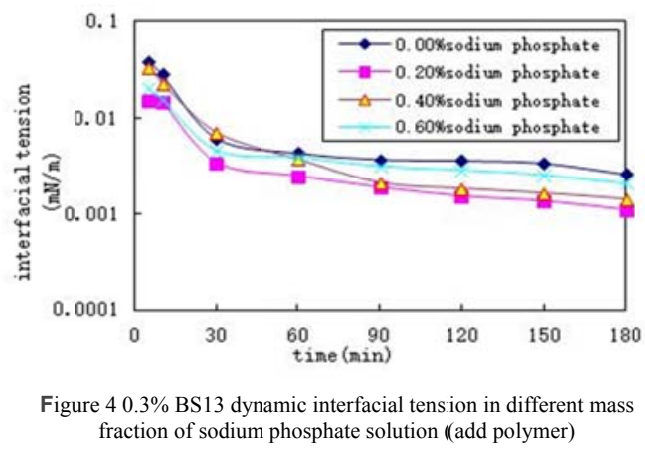

fraction of sodium phosphate solution (add polymer)

$0.3 \%$ BSA dynamic interfacial tension of the surfactant, sodium phosphate solution as shown in figure $3,0.3 \%$ BSA, adding a small amount of polymer surfactant - sodium phosphate system $(0.15 \%$ mass fraction) of dynamic interfacial tension as shown in figure 4. By figure 3 shows, because of the addition of sodium phosphate, $0.3 \% \mathrm{BSA}$, the dynamic interfacial tension of surfactant system becomes lower, the speed of up to $10-3 \mathrm{mN} / \mathrm{m}$ ultra-low interfacial tension becomes faster, and when it comes to an ultra-low interfacial tension of system, sodium phosphate optimum mass 
fraction value is $0.2 \%$. Contrast figure 3 and figure 4 , you can see that adding polymer of BSA surfactant - sodium phosphate system can still achieve ultra-low interfacial tension, but delayed in ultra-low interfacial tension of the time, and as the growth of the action time, joined the polymer interfacial tension constant and steady decline, and it takes a long time to maintain ultra-low interfacial tension, to achieve better effect of wash oil in the process of oil displacement.

2.4 The influence of temperature on the interfacial tension of BSA system or binary system separately

The influence of temperature on the interfacial tension of BSA system or binary system separately is shown in figure 5 . For binary systems, the $35 \sim 50$ degrees temperature, the impact on the interfacial tension is not big. With the temperature increasing, BSA system alone makes further reduce the interfacial tension; When the temperature reaches 55 degrees, interfacial tension can be to a low value. This shows that the temperature increases, the BSA interfacial activity increased.

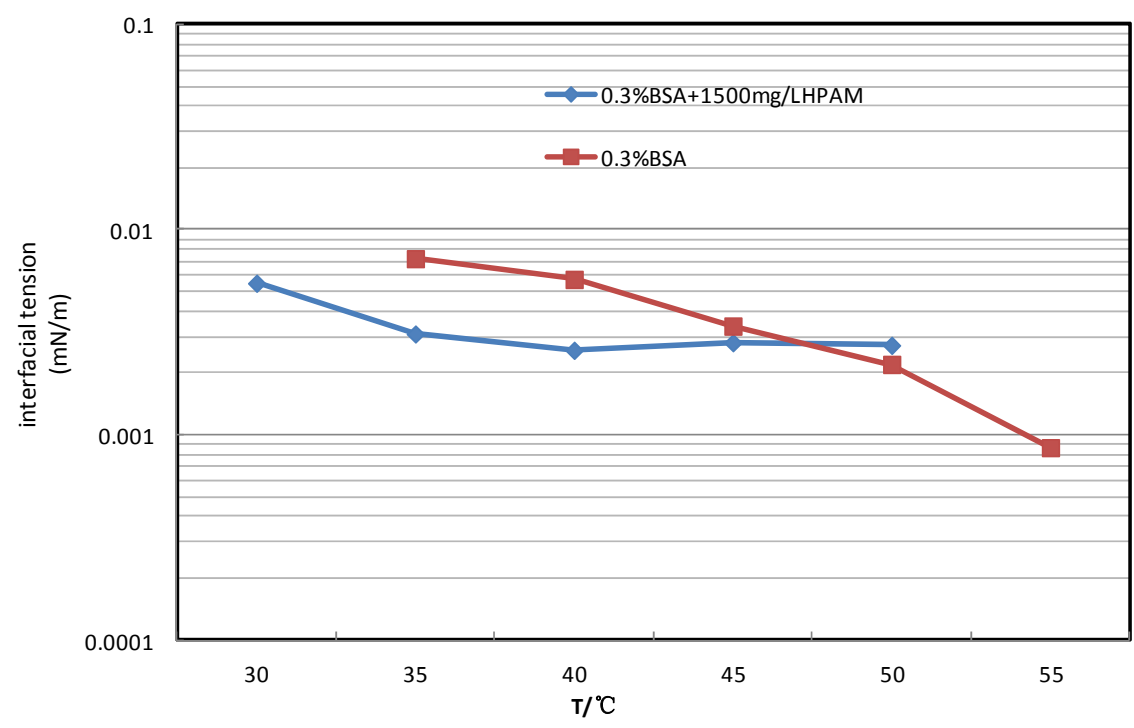

Figure 5 The influence of temperature on the interfacial tension of system

2.5 The influence of salinity and salt concentration on the interfacial tension of BSA system or binary system separately

The influence of salinity and salt concentration on the interfacial tension of BSA system or binary system separately is shown in figure 6. For binary systems with salinity increasing, the interfacial tension decreased. Separate BSA system in the salt concentration of $0 \sim 10000 \mathrm{mg} / \mathrm{L}$, had a little effects on interfacial tension. 


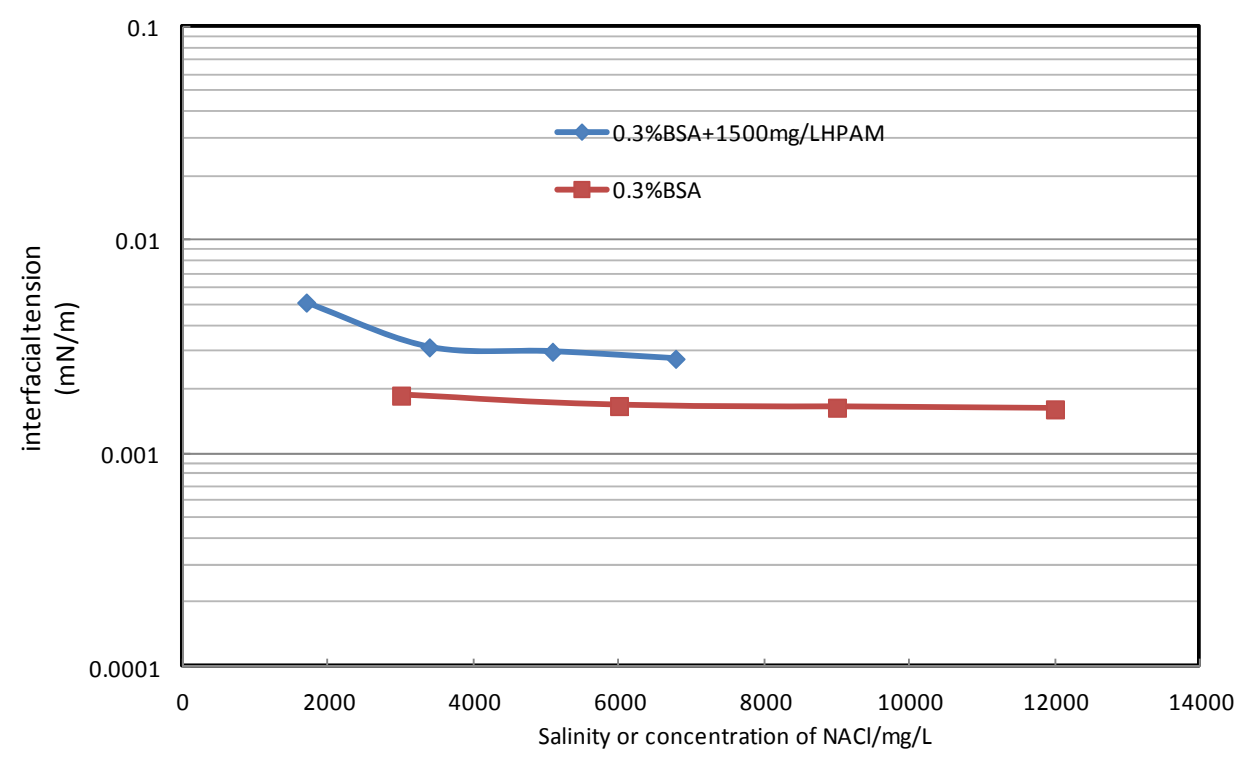

Figure6 The influence of salinity or salt concentration on the interfacial tension

\section{Conclusion}

(1) Synthesis of a new type of BSA, betaine surfactant can effectively reduce the interfacial tension, change the wettability of rock surface, improve the water phase permeability, in the effective concentration of $0.3 \% \sim 0.001 \%$ of alkaline conditions, with daqing crude oil in ultra-low interfacial tension reached;

(2) With the increase of mass fraction of BSA, non-alkali BSA system reduce the interfacial tension, in the case of BSA mass fraction is very low, it can make the system in ultra-low interfacial tension reached, at the same time that the mass fraction of ultra-low interfacial tension becomes wider.

(3) Add a small amount of sodium phosphate interfacial tension become lower or make into 10-3 $\mathrm{mN} / \mathrm{m}$ ultra-low interfacial tension speed faster, but to join the amount of sodium phosphate has a best value $0.2 \%$. Sodium phosphate is one of the most common surfactants sacrificial agent, this makes the wider scope of application of the surfactants

(4) In BSA surfactant - sodium phosphate in the binary system, adding a small amount of polymer system still can achieve ultra-low interfacial tension, and in a state of steady decline, remain at ultra-low interfacial tension for a long time, can make the system achieve better in the process of oil displacement effect of wash oil.

(5) Salinity and bivalent ions have less effect on the interfacial tension of system, can be more widely used in oilfield

\section{Reference}

[1] Yang zhenyu, Zhou hao, Jiang Jiang, etc. The performance of surfactant compound flooding in daqing oil field and the development direction of [J]. Journal of fine chemical industry, 2005, 22 (S1) : 22, 23.

[2]Li Mengtao, Liu Xiangui, Yang Xiaojun. Non-alkali binary compound system oil displacement test study [J]. Oil drilling technology, 2004, 26 (5) : 73-76.

[3] Cheng Jiecheng, Wang Demin, Li Qun and so on. Field test dynamic characteristics of asp flooding in daqing oil field [J]. Journal of oil, 2002, 23 (6) : 37 to 40.

[4] Wang Demin, new technologies to ensure continuous and stable development of daqing oilfield (bottom) [J]. Journal of daqing petroleum geology and development, 2001, (8) : 1. 
[5] Wang Demin,Cheng Jiecheng, Wu Junzheng, et al.An alkaline/surfactant/polymer field test in a reservoir with a long-term 100\%watercut [R].SPE 49 018,1998.

[6] Liu Liping, Yang Jianjun. Polyethylene/table binary recognition system performance research [J]. Journal of fault block oil and gas fields, 2004, 11 (4) : 44, 45.

[7] Sun Xinchun ,Xiang Xiangxing. Naphthenic acid salt as a surfactant in ASP flooding system research [J]. Journal of petroleum science and technology in xinjiang, 1997, 7 (2) : 5459.

[8]Li Ganzuo, shen qiang, Zheng Lijiang. New oil displacement with natural mixed carboxylate surfactant [J]. Journal of oilfield chemistry, 1999, 12 (1) : 57.

[9] Huang Hongdu. Preparation of products directly from the vapor phase oxidation of alkane (sulfonated) oil displacement with surfactant [J]. Journal of oilfield chemistry, 1987, 4 (3) : 191. 